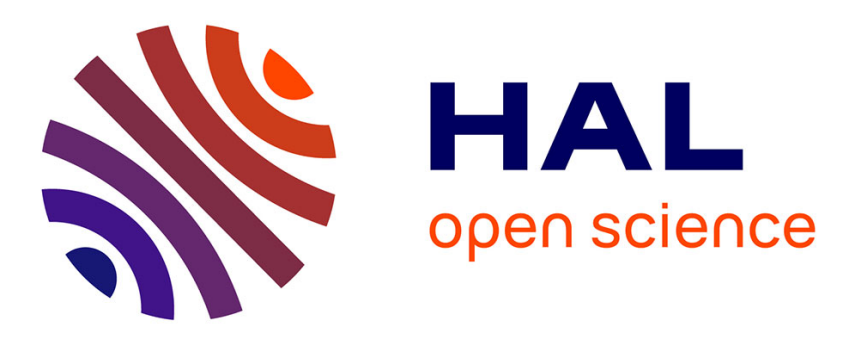

\title{
Laos. Prospections 1999 au sud de Vat Phou (province de Champassak) : du Houay Khamouane à la frontière cambodgienne
}

Marielle Santoni, Christine Hawixbrock

\section{- To cite this version:}

Marielle Santoni, Christine Hawixbrock. Laos. Prospections 1999 au sud de Vat Phou (province de Champassak) : du Houay Khamouane à la frontière cambodgienne. Bulletin de l'Ecole française d'Extrême-Orient, 1999, 86, pp.394-405. 10.3406/befeo.1999.3423 . halshs-02549150

\section{HAL Id: halshs-02549150 \\ https://shs.hal.science/halshs-02549150}

Submitted on 21 Apr 2020

HAL is a multi-disciplinary open access archive for the deposit and dissemination of scientific research documents, whether they are published or not. The documents may come from teaching and research institutions in France or abroad, or from public or private research centers.
L'archive ouverte pluridisciplinaire HAL, est destinée au dépôt et à la diffusion de documents scientifiques de niveau recherche, publiés ou non, émanant des établissements d'enseignement et de recherche français ou étrangers, des laboratoires publics ou privés. 


\section{Laos. Prospections 1999 au sud de Vat Phou (province de} Champassak) : du Houay Khamouane à la frontière cambodgienne Marielle Santoni, Christine Hawixbrock

\section{Citer ce document / Cite this document :}

Santoni Marielle, Hawixbrock Christine. Laos. Prospections 1999 au sud de Vat Phou (province de Champassak) : du Houay Khamouane à la frontière cambodgienne. In: Bulletin de l'Ecole française d'Extrême-Orient. Tome 86, 1999. pp. 394-405; doi : https://doi.org/10.3406/befeo.1999.3423

https://www.persee.fr/doc/befeo_0336-1519_1999_num_86_1_3423

Fichier pdf généré le 08/02/2019 
Laos

\section{Prospections 1999 au sud de Vat Phou (province de Champassak) : du Houay Khamouane à la frontière cambodgienne}

\section{Objectifs de la mission}

Dans le cadre du Projet de recherche en archéologie lao (PRAL), codirigé par Marielle Santoni (CNRS, UMR 9993) et Viengkèo Souksavatdy (directeur de l'archéologie auprès du ministère de la Culture laotien $)^{4}$, la prospection de l'ensemble de la partie sud de la province de Champassak (sud du Laos), qui avait été amorcée en $1998^{5}$, a été poursuivie en 1999.

Ce programme était à l'origine centré sur l'étude du site khmer monumental et religieux de Vat Phou ainsi que sur celle d'une ville ancienne sise au pied du temple, en bordure du Mékong. On sait par des inscriptions que cette ville, nommée Kurukșetra, a été fondée à la fin du $\mathrm{V}^{\mathrm{e}}$ siècle et qu'elle eut un rôle politique majeur au $\mathrm{VI}^{\mathrm{e}}$ et au $\mathrm{VII}^{\mathrm{e}}$ siècle, en tant que première capitale du Chenla, avant l'établissement d'une seconde capitale à Sambor Prei Kuk (Cambodge).

Les précédentes campagnes de fouilles (1991 - 1998) dans la ville ancienne ont permis de dégager de nombreux monuments qui confirment son ancienneté, dont le plus remarquable est un stüpa double entouré d'une douve ${ }^{6}$. Cette ville constitue un excellent référentiel pour cette période et le territoire qui s'articule autour - l'État du Chenla - est donc exemplaire pour l'évolution du monde khmer ancien, d'autant qu'il est celui qui a vu naître le premier pouvoir politique khmer. Les prospections entreprises ont pour but de découvrir ce territoire, de comprendre comment il était organisé, et quelles étaient ses relations avec sa capitale et les régions voisines.

\section{Résultats de la mission}

La région d'étude forme un triangle fermé à l'ouest par la frontière thaillandaise, au sud par la frontière cambodgienne, à l'est par le Mékong, au nord par le Houay Khamouane, une rivière qui prend sa source dans la montagne derrière Vat Phou et se jette dans le Mékong à une trentaine de kilomètres au sud (fig. 1).

Cette région, largement dépeuplée à cause des conflits incessants, n'est accessible aux étrangers que depuis peu. Certaines zones à proximité des frontières en demeurent encore interdites, en particulier une large partie de la zone sud, toujours minée. Il n'existe aucune carte très récente et les routes indiquées sur les deux séries de cartes anciennes, par ailleurs souvent inexactes, n'existent plus. La seule piste praticable, tracée récemment, n'est pas encore cartographiée. L'essentiel de la préparation de la prospection s'est donc fait à partir de photographies aériennes de 1952 et de 1981, sur lesquelles sont nettement visibles la grande chaussée ancienne menant de Vat Phou à Angkor, les grands réservoirs khmers, et

4. Le PRAL, qui a vu le jour en 1991 sur un financement du ministère des Affaires étrangères français, est un projet de coopération lao-français en archéologie entre la direction de l'Archéologie et des Musées de la RDP Lao, ministère de la Culture (Vientiane), et le musée national des arts asiatiquesGuimet (Paris). Huit campagnes de fouilles et de prospections ont déjà eu lieu depuis 1991.

5. Voir M. Santoni et Ch. Hawixbrock, BEFEO, 85 (1998), p. 387-405.

6. Ibid. 
les plus grandes des structures monumentales, ainsi que, parfois, de larges enceintes correspondant peut-être à des habitats anciens et des zones de parcellaire orienté est-ouest apparaissant sous le parcellaire récent.

Mis à part ceux en bordure du Mékong, la plupart des sites se trouvent en forêt et sont d'accès difficile; ils nous ont été signalés soit par les militaires, soit par les bûcherons qui les ont découverts. Ils sont presque toujours associés à un réservoir ancien, qui, même de petite taille, peut être repéré a posteriori sur les photographies aériennes.

$\mathrm{Au}$ total, une vingtaine de sites dont quinze comportant un monument ont été ainsi inventoriés et situés grâce au croisement des données fournies par le GPS, les cartes et les photographies aériennes. Chacun a fait l'objet d'une fiche d'inventaire, d'un relevé, de photographies et d'un enregistrement en vidéo. Une cinquantaine de pièces d'eau / mares / réservoirs accessibles ont été également répertoriés. Mais il faudrait une recherche systématique pour déterminer s'ils sont anciens ou non, et savoir à quel type de structure non monumentale ils se rattachent.

Il faut enfin préciser que toutes les structures monumentales ont été détruites à des degrés divers. Toutes ont été creusées au centre par les pillards à la recherche des dépôts de fondation. Certaines sont encore debout, d'autres écroulées, certaines enfin éventrées et éparpillées.

A l'issue des deux saisons de prospections, il semble que l'on puisse répartir ces sites en plusieurs groupes ou ensembles :

\section{a) Les temples des villages le long du Mékong}

That Don Sai, Non Phakao, Non Sombat, Phon Douk, Ban Saphang (fig. 1, $\mathrm{n}^{\circ} 1$ à 4 et 6)

Par le passé comme de nos jours, le Mékong est source de richesse pour les riverains. Les villages modernes se superposent aux anciens. L'occupation est naturellement plus dense qu'à l'intérieur des terres, et s'étend de façon presque continue le long des rives.

Plusieurs installations ont été repérées, dont certaines possèdent un ou plusieurs sanctuaires de briques ou de latérite avec des linteaux richement sculptés. Le plus souvent, seuls les linteaux et autres éléments architecturaux de grès (encadrements, marches) demeurent, avec un peu de mobilier cultuel (piédestaux, linga, fragment de stèle inscrite) : les temples ont été soit remaniés (Ban That, sur l'île de Don Sai, a été reconstruit au début $\mathrm{du} \mathrm{XX}^{\mathrm{e}}$ siècle avec les matériaux anciens pour être transformé en stüpa), soit rasés récemment pour permettre la mise en culture des terres, le passage d'une route, la construction de maisons (Non Phakao, Non Sombat, à Ban Mai). Les linteaux (à arc simple et médaillon central, avec ou sans makara) sont du type 1 de la période préangkorienne (fig. 2). Un seul petit sanctuaire (Phon Douk à Ban Veune Khene) possède un linteau à feuillage de style " Prei Kmeng-Kompong Prah », un peu plus tardif (apporté au musée de Paksé). Les autres vestiges de surface, finalement assez rares, sont des meules khmères (pesani) avec leur rouleau et quelques tessons (associés à Ban Saphang à des fragments de briques et des graviers). On note aussi la présence isolée d'un linga de style assez fruste, trouvé dans les rapides entre ce village et l'île d'en face, Don San. Sur l'île de Khong, plus au sud, nous avions aussi repéré en 1992 les vestiges d'un sanctuaire avec linteau décoratif.

Le fait que seuls les sites du bord du Mékong aient livré des linteaux sculptés, qui plus est de grande qualité, et que les sanctuaires soient parfois très rapprochés, nous incite à penser que ces bourgades étaient plus peuplées et plus riches que les villages de l'intérieur des terres, comme c'est encore le cas maintenant. Pour le moment, aucun monument angkorien n'a été trouvé, mais des vestiges mineurs confirment la continuation de l'occupation pendant la période angkorienne. On peut penser que les temples 
préangkoriens ont été utilisés et entretenus pendant une assez longue période. Cette partie de la province n'a peut-être pas fait l'objet de campagnes de construction pendant la période angkorienne.

\section{b) Les petits temples préangkoriens en latérite, à l'intérieur des terres}

Ban Kouay, Nong Sombat Nai, Nong Sombat Nyai, Nong Sombat Noi, Nong Saming, Houay Kadienne (fig. $1, \mathrm{n}^{\circ} 5,9,10,12,13,15$ )

À l'intérieur des terres, entre les grands axes de circulation, cinq petites structures très détruites et pillées ont été découvertes. Aucune d'entre elles ne comportait de linteaux décorés. En revanche, trois des cinq structures conservaient une inscription. Il semble que l'on avait là un peuplement agricole moyennement dense, regroupé en petits villages (dont Ban Kouay, avec quelques traces d'occupation, blocs de grès et de latérite, pourrait être un exemple), caractérisés par la présence d'une multitude de petites pièces d'eau.

Ce sont de petits sanctuaires de 7 à $8 \mathrm{~m}$ de côté, massifs, construits en blocs de latérite de petite taille, qui sont pratiquement du même module que les briques des sites préangkoriens ${ }^{7}$. Ils comprenaient quelques éléments en grès, tels qu'encadrements de portes, marches de seuils et, dans un cas, colonnettes. Les linteaux n'ont pas été retrouvés. Il est vrai qu'ils ont pu être emportés par des pillards, mais l'allure générale des constructions évoque plutôt un type de structure assez simple, sans décor très élaboré. Quatre des cinq temples ouvrent à l'est et sont associés à un ou plusieurs baray de taille moyenne, dont le principal est aussi placé à l'est. Un des temples (Nong Sombat Noi) est inachevé, les éléments lapidaires ne sont pas décorés. Un autre (Nong Saming) est entouré d'une douve. Seul Nong Sombat Nyai s'ouvre vers l'ouest, et est pourvu d'une enceinte à gopura en latérite ; son baray se trouve au nord. Un fragment d'inscription le rattache à la période de Jayavarman I ( $\mathrm{VII}^{\mathrm{e}}$ siècle). D'un autre sanctuaire (Nong Sombat Nai) provient une stèle inscrite, de forme inhabituelle (longue et étroite), à peu près illisible (épaufrée, caractères en surcharge), probablement de la même époque, et peut-être antérieure. Le cinquième temple enfin, le plus important (Houay Kadienne), comprend un sanctuaire principal et un sanctuaire annexe ${ }^{8}$. Sur les deux tableaux de grès de l'encadrement de porte est gravée une longue inscription en sanskrit et en khmer, qui précise exactement la date de fondation (18 mai 654) sous le règne de Jayavarman et donne le nom du fondateur, un notable qui se dit habitant de Kuruksetra (Claude Jacques, communication personnelle). Ceci revêt une importance toute particulière, car la ville ancienne de Vat Phou est, à sa fondation par Devānika au milieu du $v^{\mathfrak{e}}$ siècle, nommée Kurukșetra. Cette inscription montre donc, d'une part que ce nom était encore conservé au $\mathrm{VII}^{\mathrm{e}}$ siècle, et d'autre part que toute la région était encore administrée à ce moment-là par la première "capitale » du Chenla, et non par la nouvelle capitale, Sambor Prei Kuk - İsānapura. La date donnée par

7. Les bancs de latérite affleurent dans tout ce périmètre et c'est peut-être pourquoi les constructeurs de ces temples l'ont utilisée de préférence aux briques, tout en respectant les modules en usage pour celles-ci, alors que la majorité des constructions en latérite postérieures à cette époque sont montées avec des blocs de grande taille.

8. Nous avions déjà visité ce site en 1998, sans pouvoir faire aucun débroussaillage (cf. $B E F E O$, 85 : 395). On voyait un sanctuaire encadré au nord et au sud par deux monticules que nous pensions renfermer deux structures. En 1999, un nettoyage de surface a permis de se rendre compte que si le monticule nord était bien une structure de latérite, le monticule sud n'était formé que de terre, rejetée par les pilleurs. Il s'agit donc plus probablement d'un temple composé d'un sanctuaire majeur accompagné au nord d'un sanctuaire mineur, ouvrant vraisemblablement à l'est et qui aurait pu abriter la parèdre de l'idole principale. Le temple semble avoir été entouré d'une simple levée de terre ayant fonction d'enceinte. 
l'inscription devient la plus ancienne connue pour le règne de Jayavarman I : elle permet d'avancer de trois ans le début de son règne. Des indications sur les dons de terres cultivées, de bétail, de personnel, sont détaillées dans une longue liste. À ce temple était associée une statue - un dvārapāla ou une divinité agenouillée - de style plus tardif, qui pourrait dater du $\mathrm{X}^{\mathrm{e}}$ siècle, ce qui montre que ce monument était encore utilisé pendant la période angkorienne (fig. 3). Le corps, d'assez grande taille, a été brisé en deux au niveau des hanches assez récemment. La tête (vendue) et les mains ont disparu, ce qui empêche son identification. Aucun décor vestimentaire n'est ornementé et le sampot, descendant jusqu'aux genoux, est lisse. Mais le cordon brahmanique est indiqué. Sa position est cependant assez inhabituelle puisque la statue est agenouillée les deux genoux en terre, position qui ne se rencontre que pour les Prajñāpāramitā de la fin du XII ${ }^{\mathrm{e}}$ siècle.

Ce type de structure est très difficile à repérer, il est vraisemblable qu'il en existe d'autres. Celles trouvées montrent une occupation déjà assez homogène sous Jayavarman I.

c) Structures isolées, sanctuaires sylvestres et carrières de grès

Nong Boua Bo Pong, Sang Mouang, carrière, Nong Sombat (fig. 1, $\mathbf{n}^{\circ}$ 7, 8, 11, 14)

Les sanctuaires ne sont pas nécessairement des monuments. Ainsi, un monticule assez élevé, qui paraît artificiel et qui est associé à un baray, a été considéré comme un site (Nong Sombat ou «mont du trésor », fig. $1, \mathrm{n}^{\circ} 7$ ). Mais, toujours à l'intérieur des terres, nous avons trouvé deux sites très intéressants. Le premier (Nong Boua Bo Pong) n'est marqué que par la présence de plusieurs baray, de monticules, et de deux piédestaux de statues isolés, témoignant d'un style de transition (préangkorien tardif ou angkorien précoce). On se trouve sans doute sur une ancienne occupation villageoise, sans monument. Les idoles pouvaient être simplement posées sous un auvent, sur un monticule de terre ou une plate-forme de bois. Un des deux piédestaux était enterré dans une fosse, dans un petit bois, mais il semble avoir été déplacé.

Le second site (Sang Mouang) est une sorte de «sanctuaire sylvestre »: une cuve à ablution de linga est finement sculptée à même un bloc de rocher émergeant du sol (fig. 5). Un autre rocher porte un motif de dépressions circulaires reliées par un réseau de petites rigoles, pour lequel nous ne pouvons proposer, à l'heure actuelle, aucune interprétation (fig. 4). Ce site se trouve dans la boucle d'une rivière, ou plutôt d'un torrent, dont le lit est formé par un chaos de blocs de grès. Un des blocs porte des traces de découpe, ce qui suggère l'utilisation du lieu comme carrière, et justifie peut-être l'installation de ce petit sanctuaire champêtre.

Une autre carrière de grès, située au milieu de rizières actuelles, et ne répondant à aucun nom, a également été repérée (fig. $1, \mathrm{n}^{\circ} 8$ ).

\section{d) Les temples de la route de Vat Phou à Angkor, le long des montagnes}

That Na Samliang, Dong That, That Ban Done, That Nang Ing (Pram Loveng), Nong Pham-Ban Vienne

De Vat Phou partait une chaussée ancienne surélevée, d'une dizaine de mètres de large et de deux à trois mètres de haut, qui menait, après un parcours de presque $300 \mathrm{~km}$ vers le sud puis vers l'ouest, à la région d'Angkor où elle se mêlait à un réseau routier plus dense.

Cette route est très nettement visible, sur presque tout son parcours, sur les photographies aériennes des années 1950 , et a été reportée en partie par les cartographes de cette époque sur des fonds de cartes des années 1930, qui malheureusement manquent par endroits singulièrement d'exactitude. La partie en territoire laotien court sur $100 \mathrm{~km}$ (de Vat Phou à la frontière cambodgienne). Actuellement, la route ancienne se confond sur 
une partie de son trajet avec un chemin moderne, et la plupart du temps n'est marquée que par des lignes d'arbres qui ont poussé dessus, préservant des buttes espacées. C'est dire qu'elle est considérablement érodée et serait difficile à repérer sans l'aide des photographies. Apparaissent également à intervalles réguliers des baray, grands réservoirs rectangulaires formés de levées de terre, mesurant environ $100 \mathrm{~m}$ x $200 \mathrm{~m}$. C'est à proximité de ces baray qu'il convient de rechercher les temples, quoique tous les baray ne soient pas nécessairement associés à un sanctuaire, et quand c'est le cas, n'en soient pas nécessairement très proches (parfois à $1 \mathrm{~km}$ de distance).

La première grande étape depuis Vat Phou, à une quinzaine de kilomètres au sud, est Ban That, un temple à trois sanctuaires en grès (inachevés) alignés sur une plate-forme surélevée et une stèle inscrite. Jusqu'à présent, c'était le seul monument connu sur cette route (mis à part les sanctuaires proches de Vat Phou et qui font partie du complexe).

En fait, les prospections nous ont permis d'en trouver au moins cinq autres, s'échelonnant sur la route ancienne (fig. 1), distants de 6 à $19 \mathrm{~km}$ environ (That Na Samliang, Dong That, That Ban Done, That Nang Ing-Pram Loveng, Nong Pham-Ban Vienne). Notons qu'il pouvait y avoir aussi des sanctuaires de très petite taille, voire en matériaux périssables, élevés sur une simple butte et qui ont pu disparaître. Précisons aussi qu'il nous a été interdit cette année d'explorer systématiquement la route ancienne tout du long, par crainte des mines.

Ces cinq temples sont tous bâtis sur le même modèle, à quelques variantes près (fig. 8) : une enceinte de latérite (mur à crête) presque carrée, d'environ $30 \mathrm{~m}$ de côté, ouvrant à l'est par un gopura, entoure un ensemble composé d'une tour-sanctuaire (plan carré d'environ $6 \mathrm{~m}$ de côté, quatre faux étages, trois fausses portes et une porte ouvrant à l'est), et de deux bibliothèques rectangulaires situées de part et d'autre de l'entrée et ouvertes à l'ouest. La tour est toujours en brique, l'encadrement de porte et le linteau toujours en grès. La tour repose toujours sur un socle de grès et un soubassement de latérite. L'accès aux entrées vraies ou fausses se fait par des escaliers (disparus ou enfouis) et des marches en accolade en grès, décorées (ébauchée à Dong That) ou non (Ban Done, fig. 6).

Les variantes sont marquées par l'emploi plus ou moins important du grès dans la construction de la tour. Il est parfois utilisé seulement pour les linteaux des fausses portes (Nong Pham), pour la base des murs, les piédroits, chapiteaux et colonnettes de la porte (Ban Done), parfois aussi, pour les fausses portes (Dong That, Na Samliang) et peut-être même les corniches (Dong That). Les murs des bibliothèques peuvent être en latérite ou en briques, la couverture est toujours en briques. La base du gopura est parfois en grès.

L'enceinte de latérite est elle-même toujours entourée d'une douve délimitée par des levées de terre. Le baray associé au temple est situé à l'est. Rectangulaire, il mesure au moins une centaine de mètres de long (sauf à Nong Pham ?). Souvent, d'autres petites pièces d'eau sont associées, et l'ensemble d'un complexe religieux peut être englobé dans une plus grande enceinte de terre, de 300 à $500 \mathrm{~m}$ de côté, qui pourrait correspondre à une enceinte de domaine. Dans un cas (Ban Done), la rivière proche a servi de carrière de grès.

Autre point commun et un des plus marquants : aucun de ces temples n'a été tout à fait achevé, du moins en ce qui concerne le décor : parfois les tympans, bases et corniches sont à peine ébauchés, encore en encorbellement, ou déjà ravalés en oblique. Dans le meilleur des cas, les moulures ont été taillées mais ne portent pas encore leur décor sculpté. Parfois l'amorce d'un décor de feuillages et flammes est visible sur les rampants des tympans (Ban Done, Na Samliang, fig. 7). Les fausses portes sont restées sans décor, les colonnettes octogonales également. Autant qu'on puisse en juger d'après les éléments lapidaires tombés, les linteaux sont aussi restés à l'état brut. Cependant tous ces temples, 
dédiés à Śiva, ont été utilisé, comme en témoignent les piédestaux à linga de style angkorien.

La présence de l'enceinte et des bibliothèques, l'aspect des faux étages, le style du mobilier cultuel, indiquent la période angkorienne. Une datation au $\mathrm{X}^{\mathrm{e}}$ siècle est probable. Il est possible que l'un de ces temples au moins (Dong That) ait remplacé un sanctuaire préangkorien (présence aux environs d'une colonnette préangkorienne).

Un seul des temples (That Nang Ing ${ }^{9}$ ) apparaît légèrement différent : les moulures de brique sont particulièrement fines, le décor du linteau et du tympan est déjà ébauché, avec des espaces en réserve, celui des colonnettes octogonales est achevé (angkorien classique), enfin un avant-corps maladroit a été ajouté. D'autre part, il est situé assez près - trop près - $(6 \mathrm{~km}$ et $12 \mathrm{~km})$ des étapes précédentes et suivantes : si l'on n'en tenait pas compte, la distance séparant les quatre autres temples serait régulièrement de 18 à $19 \mathrm{~km}$. Il pourrait s'agir du réaménagement d'une fondation antérieure, ou bien d'une fondation sur un village particulièrement important. Des traces de parcellaire ancien ont été repérées sur les photographies aériennes, et les baray (dont les fameux « cinq compartiments ») sont particulièrement nombreux dans la zone.

La construction rapide de ces temples, quasiment sur le même modèle, leur échelonnement le long de la route et leur état d'inachèvement semblent témoigner d'une volonté politique - qui n'a peut-être pas pu se maintenir - de relier Angkor à Vat Phou. Après une période sinon d'oubli, du moins de repli de la province, ce site reprend de l'importance comme lieu d'origine de la première dynastie de rois khmers, une manière pour ces rois d'Angkor d'affirmer la légitimité de leur pouvoir sur l'ensemble du royaume, qui comprend à ce moment-là non seulement le Cambodge, mais une bonne partie du nord-est de la Thaïlande et le sud du Laos.

La densité de l'occupation préangkorienne dans cette zone reste à évaluer : était-elle simplement faible, ou bien a-t-elle été oblitérée par l'occupation angkorienne? La présence, non loin des temples, et en tous cas tout le long de la route, de très grands baray se justifie-t-elle par une expansion de population et une intensification de la mise en culture à la période angkorienne ? Ou bien simplement ont-ils été creusés là pour le confort des voyageurs?

Sur toute la zone de prospection, la distribution des réservoirs reste à étudier. Les plus grands (100 à $200 \mathrm{~m}$ ) semblent être en rapport avec les routes (ainsi, il existerait une route transversale est-ouest, du Mékong aux montagnes et à la chaussée Vat Phou - Angkor). Ceux de taille moyenne $(50$ à $100 \mathrm{~m})$ sont souvent directement associés à des temples, mais peuvent aussi appartenir à une installation villageoise ancienne. Les petits (moins de $50 \mathrm{~m}$ ), qui foisonnent, peuvent correspondre à de petits groupes d'habitations isolées, aussi bien anciens que modernes.

\section{Interprétation et chronologie}

Nous avons dû, au cours de ces premières prospections, aller à l'essentiel. La difficulté d'accès aux sites, le peu de temps et surtout le peu de liberté d'action qui nous ont été

9. Visité une première fois en 1998 , voir $B E F E O, 85: 396$ et photo 10 . Il s'agit très probablement du Prasat Pram Loveng («tour aux cinq compartiments » en khmer) qui avait été mentionné dès 1901 par É. Aymonier (Le Cambodge, vol. 2, Paris, Leroux, p. 181) et figurait sous deux numéros différents dans E. Lunet de Lajonquière, Inventaire descriptif des monuments du Cambodge (t. 2, Paris, 1907, p. 61, $n^{\circ} 325$ et p. 73, $n^{\circ} 337$ ), mais dont la localisation était incertaine et que la description imprécise, visiblement de seconde main, ne permettait pas de reconnaître. Nous avons pu finalement l'identifier cette année à That Nang Ing en retrouvant à proximité les «cinq mares » orientées nord-sud décrites dans l'inventaire : elles portent le nom lao de Ha Hong, « cinq chambres ». 
laissés ne permettaient pas d'explorer systématiquement le terrain, en particulier pour trouver des traces fugaces d'installations villageoises ou de sites préhistoriques. Pour le moment, nous devons nous contenter des indications incomplètes (tout dégagement étant interdit) fournies par la présence des monuments, sachant que nous sommes loin de les avoir tous vus. Les datations que nous attribuons aux structures sont relativement incertaines (les inscriptions ne datent qu'elles-mêmes, des éléments ont pu être réemployés). L'interprétation qui suit sera sans doute à corriger.

$\mathrm{Au} \mathrm{VII}^{\mathrm{e}}$ siècle, l'intérieur des terres est occupé par de petits villages dont certains ont un temple, assez simple, peu décoré, construit en latérite. Des inscriptions de la période de Jayavarman I y sont souvent associées. Le plus important d'entre eux est Houay Kadienne qui comprend deux sanctuaires et cinq baray, ainsi qu'une très longue inscription qui permet de mettre la région directement en relation avec la ville ancienne de Vat Phou, à $50 \mathrm{~km}$ au nord. Mais dans l'ensemble, les temples paraissent modestes. Au moins l'un d'entre eux n'a jamais été achevé. On compte aussi des occupations de moindre importance avec des sanctuaires non monumentaux. Certaines activités artisanales sont bien attestées (carrières de grès).

À la même époque, quelques villages sur le Mékong ou dans les îles sont suffisamment prospères pour faire élever des sanctuaires apparemment plus importants, et les décorer richement.

$\mathrm{Au} \mathrm{VIII}^{\mathrm{e}}$ siècle, les campagnes de construction semblent se ralentir, reflétant peut-être l'insécurité du royaume. Seul Phon Douk (Ban Veune Khene), en bordure du Mékong, pourrait dater de cette période (d'après le style du linteau).

$\mathrm{Au} I \mathrm{I}^{\mathrm{e}}-\mathrm{X}^{\mathrm{e}}$ siècle commence probablement la construction de la chaussée ancienne (Angkor - Vat Phou) et de la série de temples qui la jalonnent (peut-être dans certains cas érigés sur un monument antérieur). Ceux-ci ne seront jamais achevés mais ont toujours été utilisés (mobilier cultuel). La construction a parfois été reprise. Les routes sont alors jalonnées de très grands réservoirs d'eau. Mais il est difficile de dire si la construction de la route a eu un impact réel sur le peuplement et le développement de la province.

En fait, les structures monumentales, peut-être étroitement liées à un souverain qui en fait financer l'érection, ne sont pas nécessairement représentatives de la répartition et de la densité de la population. Tout au plus peut-on penser que le pouvoir politique était fort dans cette région, au $\mathrm{VI}^{\mathrm{e}}-\mathrm{VII}^{\mathrm{e}}$ siècle, période de domination du royaume du Chenla, issu de Vat Phou. À l'époque de Jayavarman I, la capitale est au sud, à Sambor Prei Kuk, mais régit toujours la région nord. Après un siècle d'isolement relatif (peut-être en l'absence d'un pouvoir central), les relations avec le reste du pays reprennent, probablement peu après la fondation d'Angkor au $\mathrm{IX}^{\mathrm{e}}$ siècle. Ceci confirme ce que l'on a observé à Vat Phou (donations, fondations religieuses et reconstructions) et dans les environs (To Mo, Khan Mak Houk). Ces relations s'intensifient au $\mathrm{XI}^{\mathrm{e}}$ siècle avec la reconstruction du sanctuaire de Vat Phou. Les remaniements et remises en état se poursuivent aux $\mathrm{XII}^{\mathrm{e}}$ et $\mathrm{XIII}^{\mathrm{e}}$ siècles avec entre autres la construction, le long de la route, d'une chapelle d'hôpital (Thao Tao), sous le règne de Jayavarman VII qui couvrira l'empire d'un réseau de routes conduisant aussi loin que Vientiane, au nord, par exemple.

\section{Conclusion}

$\mathrm{Du}$ point de vue historique, les diverses inscriptions trouvées depuis le début de nos travaux confirment que la région est bien le lieu d'origine de la dynastie fondée par Mahendravarman, probablement né lui-même vers Vat Phou dont la ville ancienne fut intensivement occupée pendant deux siècles au moins, de la fin du $\mathrm{v}^{\mathrm{e}}$ siècle (fondation, stèle de Devānīka) à la fin du $\mathrm{VII}^{\mathrm{e}}$ siècle. Il est peu vraisemblable que la ville ait eu un rôle 
crucial au-delà de cette date. Probablement déjà capitale régionale au temps de Viravarman, père de Mahendravarman, elle le demeure sous une partie du règne de ce dernier, et perd sans doute de son importance lorsqu'il prend possession des domaines de son frère, Bhavavarman, dont la capitale, Bhavapura, était probablement à Sambor Prei Kuk (İsāānapura) où régnera son fils, İsānavarman I. Le pouvoir se transporte alors vers le sud et il semble qu'après le règne de Jayavarman I, la région d'origine de la famille de Mahendravarman (sauf le sanctuaire de Vat Phou) soit négligée et les territoires qu'il prétend avoir conquis vers le nord (cf. ses nombreuses inscriptions retrouvées en Thaïlande, dans les provinces de Khorat, Khon Khaen, Surin, Prachinburi et Ubon), abandonnés des rois khmers, puisqu'on n'y a retrouvé aucune autre inscription avant celle d'Indravarman I, plus de deux siècles et demi plus tard.

Il se confirme donc que la ville ancienne de Vat Phou (qu'il faudra peut-être appeler soit Kurukșetra soit Lingapura, noms plusieurs fois mentionnés), première capitale préangkorienne, est un exemple unique et que sa « durée de vie » fut assez brève, quoique intense, avec la construction de plus d'une trentaine de sanctuaires, dont certains, comme le double stüpa de Nong Vienne, illustrent une pratique du bouddhisme très ancienne et, semble-t-il, assez originale.

Toute la province au sud de Vat Phou semble également avoir connu un grand développement, en particulier sous le règne de Jayavarman I. Pendant tout le $\mathrm{VII}^{\mathrm{e}}$ siècle, les relations avec Sambor Prei Kuk (İsānapura) étaient sans doute encore très vivaces. Au $\mathrm{VIII}^{\mathrm{e}}$ siècle, période de troubles et peut-être de domination étrangère, la province semble avoir été abandonnée à elle-même. Ce n'est qu'après la fondation d'Angkor au IX $\mathrm{X}^{\mathrm{e}}$ siècle et le raffermissement du pouvoir des rois khmers que les relations reprennent, soutenues par une volonté politique apparemment assez forte de relier - pour le légitimer - le nouveau pouvoir émergeant à son berceau, Vat Phou. En effet, des raisons purement " économiques » ou du moins commerciales ne justifient pas l'établissement de la grande chaussée, avec la construction de tous ces temples d'étapes, alors que le Mékong reste la voie de communication privilégiée. De plus, elle traverse une région assez peu fertile et difficile à irriguer, peut-être assez peu peuplée. Manque de moyens, changement d'orientation politique, ou négligence, les temples n'ont jamais reçu leur décoration finale.

Fouilles et prospections apportent en tous cas quantité d'éléments nouveaux pour la connaissance d'une période qui n'était jusqu'à présent documentée que grâce aux sources chinoises anciennes. Au-delà des informations sur l'histoire, l'art et l'architecture, les techniques, le programme de recherches devrait livrer, à long terme, des données essentielles sur l'organisation sociale urbaine et ses origines, et sur les modifications apportées à l'environnement par les activités agricoles et le développement de la distribution de l'eau, si importante par la suite dans l'empire khmer.

Marielle SANTONI (CNRS, UMR 9993) et Christine HAWIXBROCK (EFEO) 


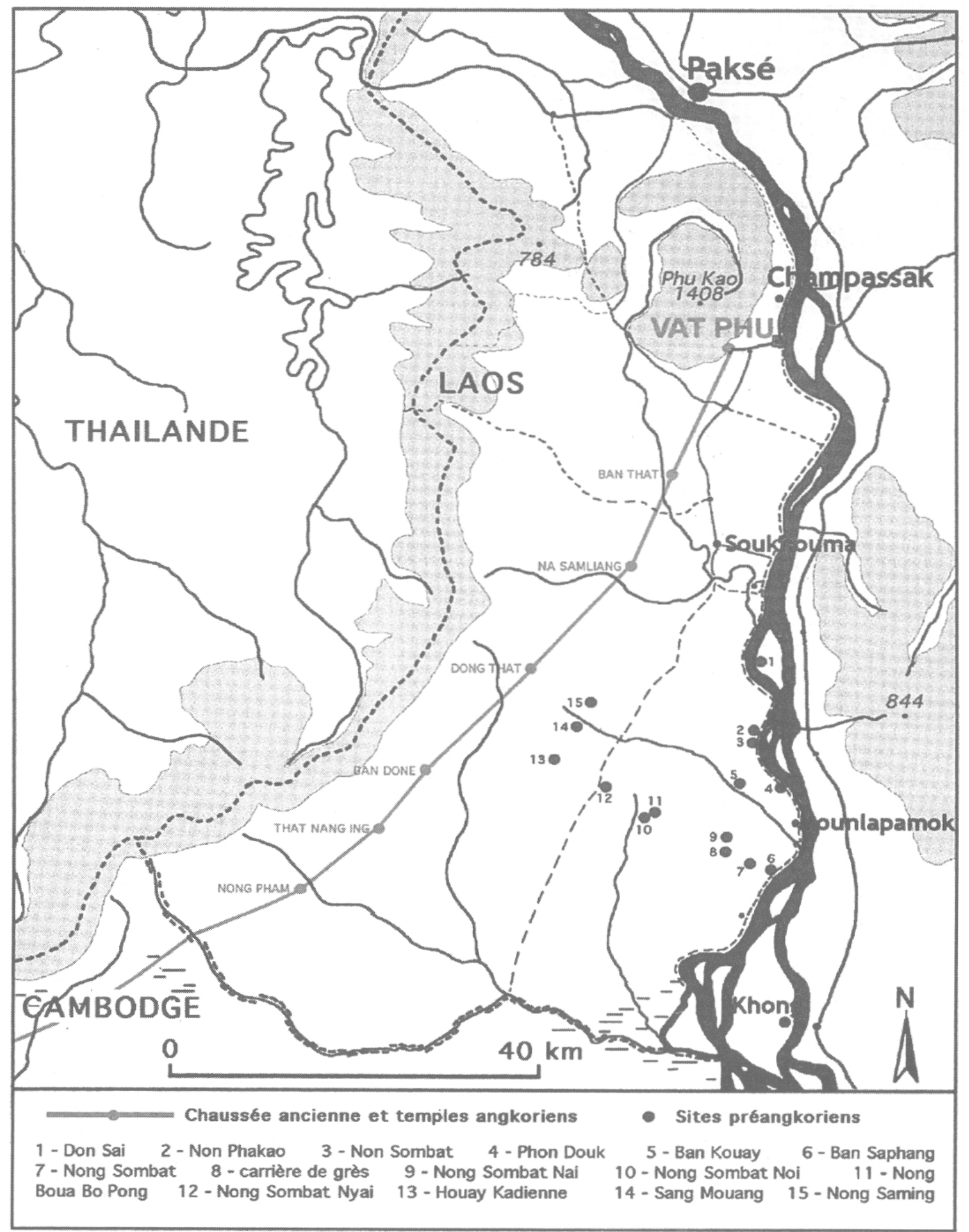

Fig. 1 - Zone de prospection dans la province de Champassak : position des sites

(Carte et photos : Marielle Santoni ; dessins : Jean-Pierre Message) 

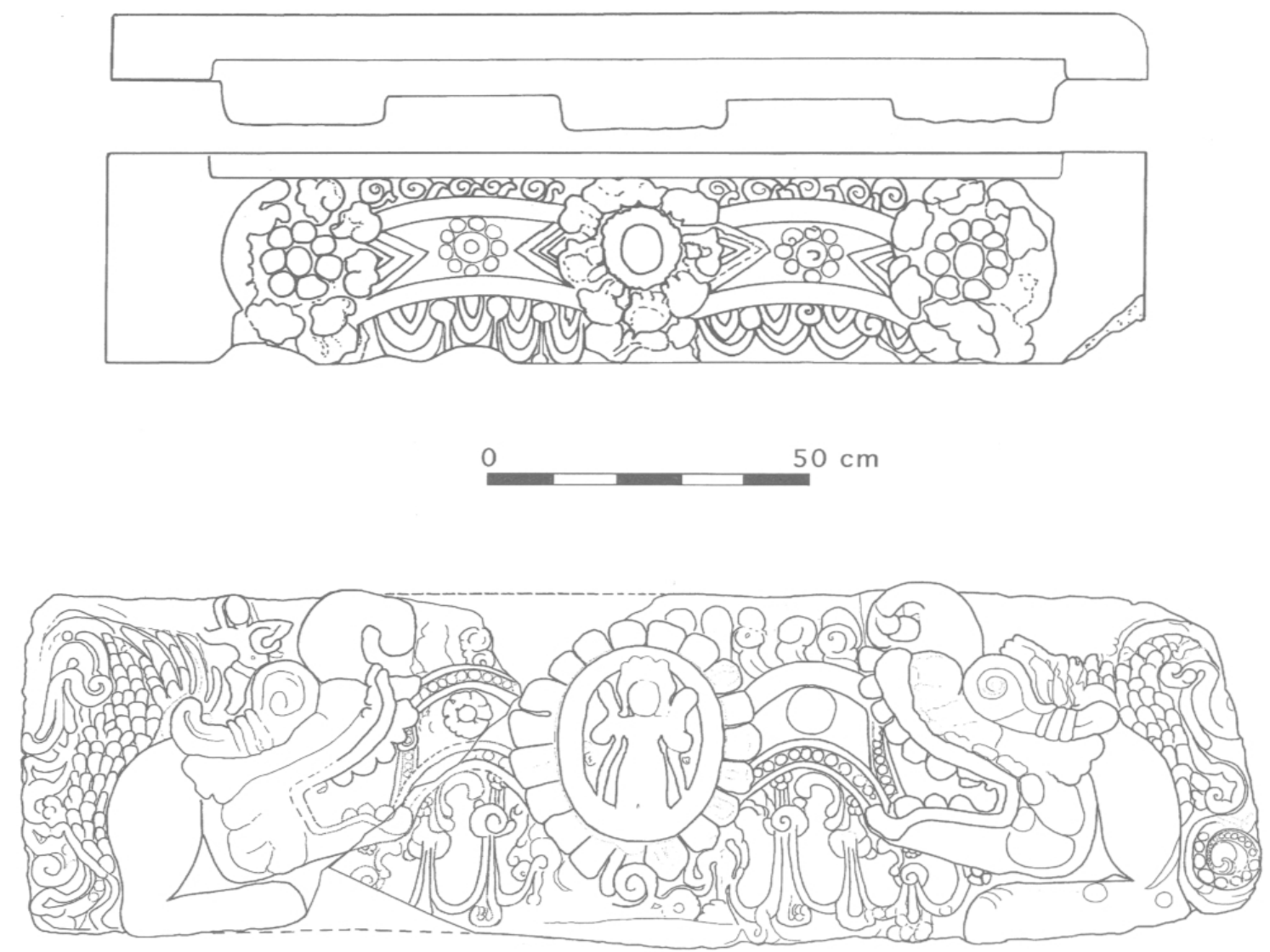

Fig. 2 - Linteaux préangkoriens : That Don Sai (en haut) et Non Sombat (en bas)

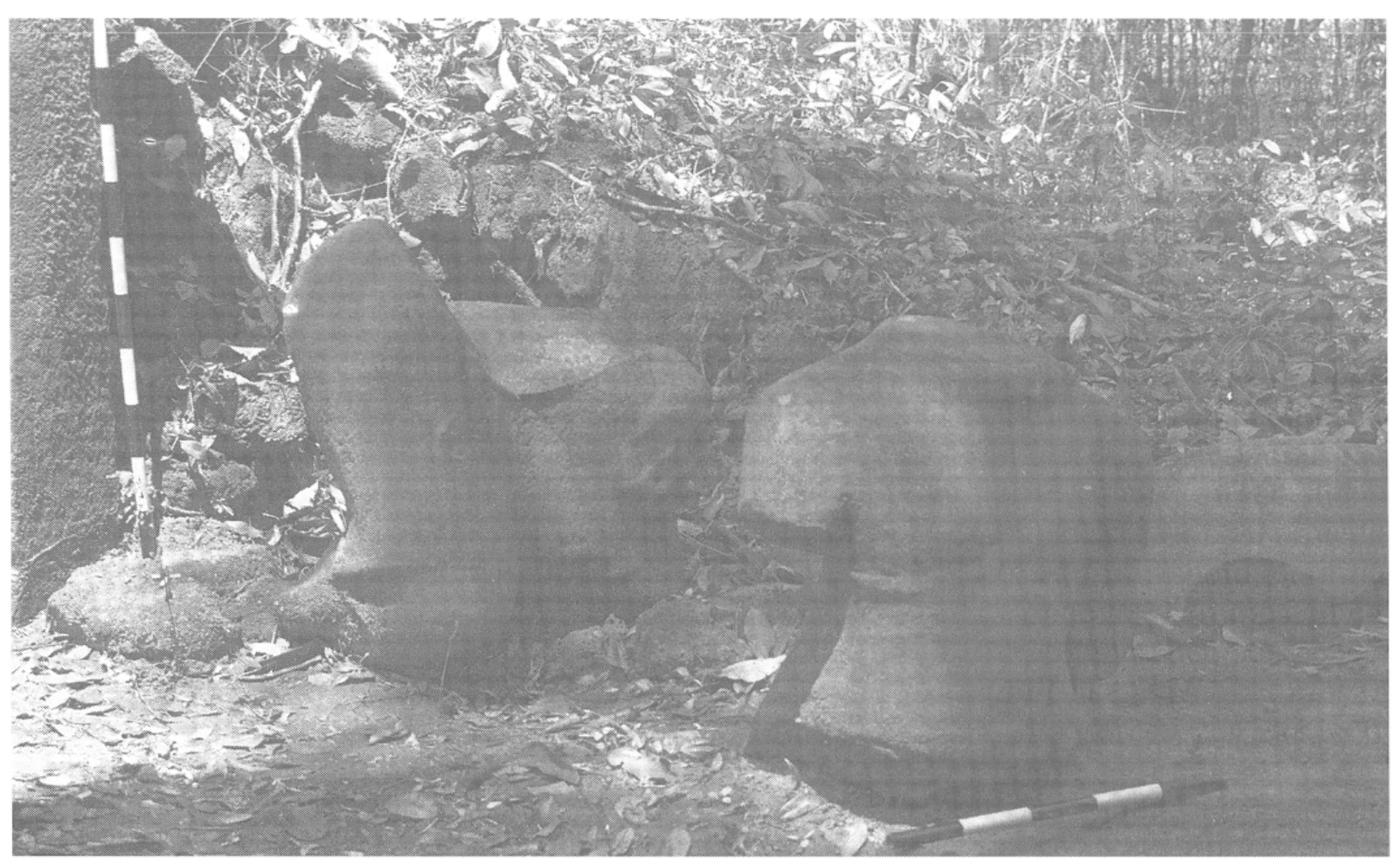

Fig. 3 - Statue brisée, devant le sanctuaire de Houay Kadienne 


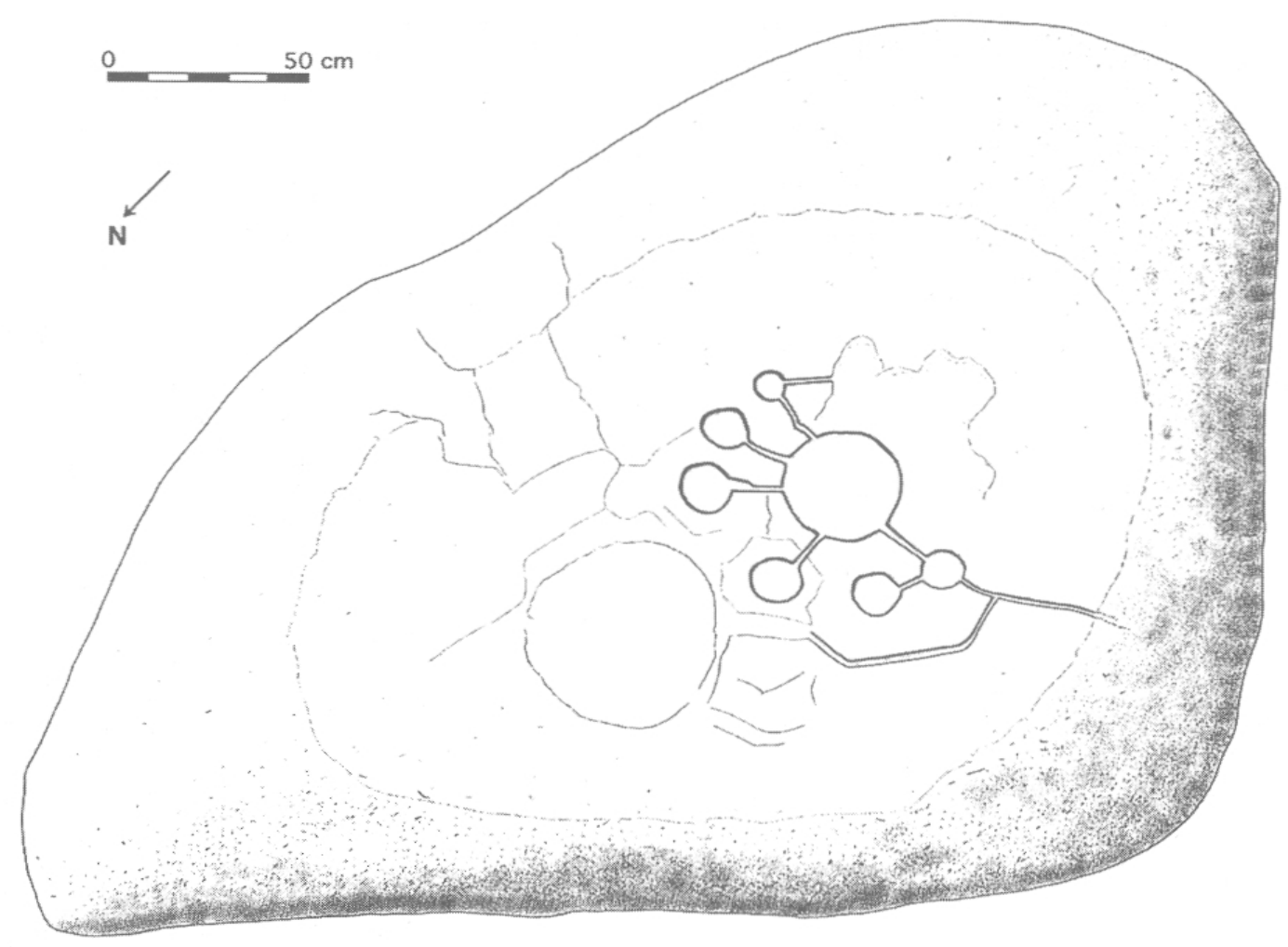

Fig. 4 - Motif en creux sur un rocher à Sang Mouang

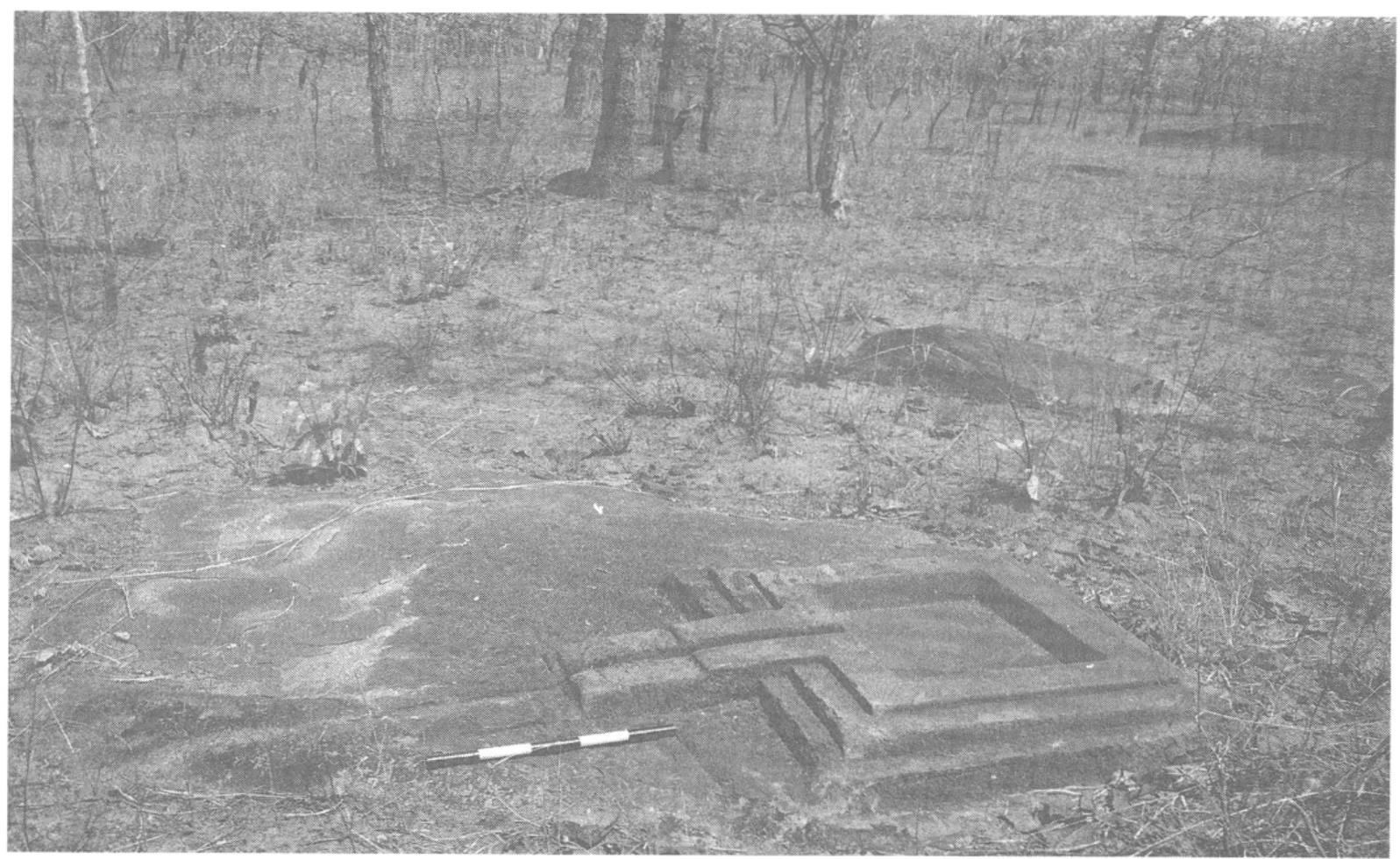

Fig. 5 - Cuve à ablution de linga sculptée sur un rocher à Sang Mouang 

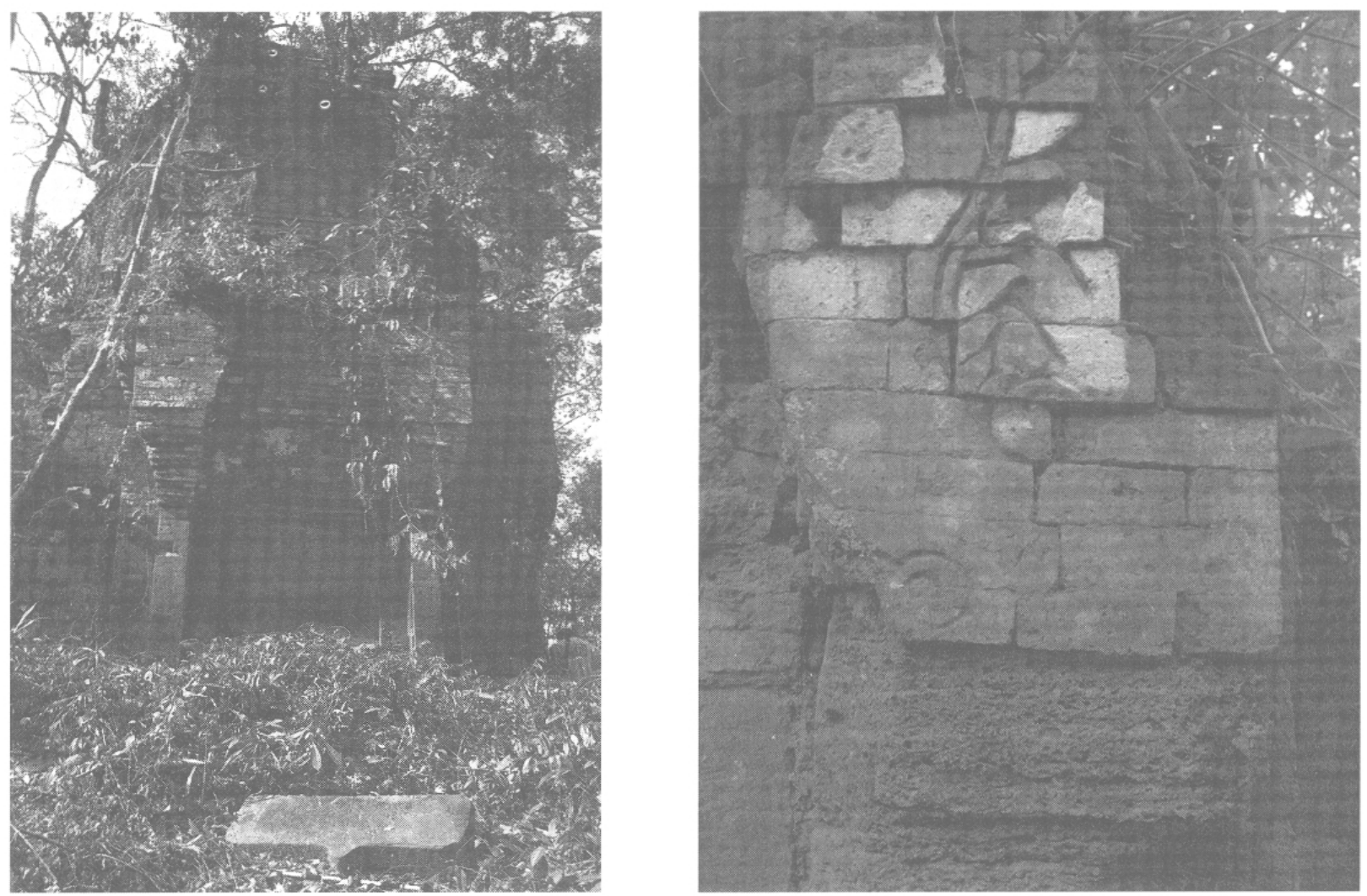

Fig. 6 - That Ban Done, face sud du sanctuaire, marche au premier plan

Fig. 7 - Décor taillé sur le rampant droit du fronton est de la bibliothèque sud de That Na Samliang

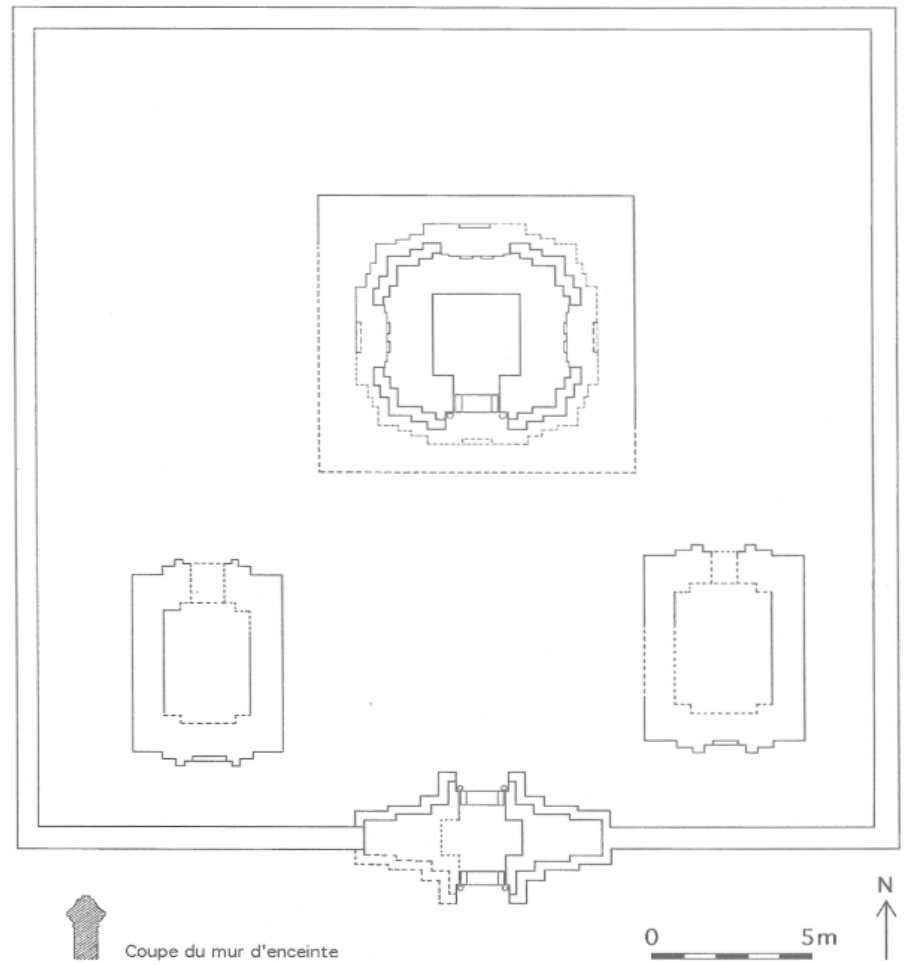

Fig. 8 - Plan de That Na Samliang 